\title{
Closing the Gap: Exploring Nurse Managers' Support to Midwives in Implementing HIV/AIDS Testing and Counselling within Protocol
}

\section{Priscilla Paulse}

https://orcid.org/0000-0002-5934-7813

University of the Western Cape,

South Africa

priscillajannet@gmail.com

\author{
Karien Jooste \\ https://orcid.org/0000-0002-6721-7293 \\ Cape Peninsula University of \\ Technology, South Africa \\ joosteka@cput.ac.za
}

\author{
Wilson Majee \\ https://orcid.org/0000-0003-1726-0979 \\ University of Missouri, United States \\ majeew@missouri.edu
}

\section{Abstract}

Midwives are expected to meet the demands of their ever-expanding roles without having a voice to contribute to the decision-making process in developing the protocols to be followed in implementing HIV/AIDS testing and counselling. The aim of the current study was to describe nurse managers' support to midwives in implementing HIV/AIDS testing and counselling within protocol at an antenatal clinic located in the Central Region of Cape Town, Western Cape, South Africa. The study followed a qualitative design to gain insight into midwives' experiences in implementing HIV/AIDS testing and counselling at an antenatal clinic and how nurse managers support midwives in this regard. In-depth individual interviews were conducted with eight $(n=8)$ purposively sampled professional midwives to understand the implementation of HIV/AIDS testing and counselling within protocol from the midwives' perspectives and field notes were taken. All the interviews were digitally recorded, transcribed verbatim and coded using the thematic approach. The study findings indicated that ongoing education, coaching and mentoring, and constructive feedback are all necessary support mechanisms for midwives to implement HIV/AIDS counselling for better client care outcomes. It was recommended that nurse managers should intensify their efforts in an antenatal clinic, supporting midwives with practice relevant information on HIV, to address the needs of pregnant women and the larger community. Well-informed

\section{UNISA $\approx$}


competent midwives are needed to apply the latest protocols and trends in midwifery practices that will uplift the health status of pregnant women in the community.

Keywords: pregnant women; midwife; antenatal clinic; HIV/AIDS protocol

\section{Introduction}

South Africa is leading the HIV/AIDS epidemic globally, with 7.1 million people living with HIV. The country also has the most comprehensive antiretroviral therapy (ART) programme in the world, with a focus on the implementation of the "test and treat" protocol and guidelines (UNAIDS 2019). However, as nurses in rural areas are the main health care professionals responsible for the implementation of policies, questions have arisen about the nursing support patients receive and their nurses' expertise regarding HIV/AIDS policies (Phetlhu et al. 2018, 876). However, the United Nations Population Fund (UNPFA 2014) states that nearly 290000 maternal deaths occur yearly, due to the lack of well-educated and regulated health workers and adequate facilities. Thus, around the world, midwives are playing an increasingly important role, not only delivering babies, but also providing other life-saving reproductive health information and services, including antenatal care, postnatal care and family planning (UNPFA 2014).

Africa has a needs-based shortage of nearly 18 million health care professionals, such as doctors, nurses and midwives (Scheffler et al. 2018,4). The shortage is up against various health challenges. HIV/AIDS and sexually transmitted infections (STIs) are common co-infections in South Africa, and constitute major public health problems (Mntlangula, Khuzwayo and Taylor 2017, 52). In South Africa, around 1.4 million HIV/AIDS infections among children were prevented between 2010 and 2018 due to the Policy and Guidelines for the Implementation of the Prevention of Mother to Child Transmission (PMTCT) Programme (Avert 2019a) and the involvement of midwives.

HIV/AIDS remains a leading source of the high mortality rates in developing countries, such as South Africa. Although various policies exist for the prevention and control of HIV/AIDS, addressing the issue is slow (Phetlhu et al. 2018, 876). Research has shown that without treatment, for a pregnant woman living with HIV/AIDS, the likelihood of mother-to-child transmission is $15 \%$ to $45 \%$. In $2017,80 \%$ of pregnant women living with HIV/AIDS were receiving ART, a significant increase from 2010 when only $51 \%$ had access to treatment (Avert 2019b).

In South Africa, the PMTCT Programme (Avert 2019a) encompasses primary prevention of HIV/AIDS; initiation of therapies; and integration of the PMTCT into maternal, child and women's health services (Mbombo and Jooste 2016). However, Tagutanazvo, Nolte and Temane $(2019,1)$ indicate the lack of midwives who refer women, following HIV/AIDS counselling and testing, for any form of further support after being tested. 
The main health professionals involved in attending to childbirth in 50 countries are midwives or nurses who attend to the majority of lower-level and higher-level facility births (Campbell et al. 2016, 2). The shortage of health care professionals in Africa is worsened by skill-mix imbalances, uneven geographic distribution, negative work environments, and a weak knowledge base (WHO 2016). In this complex situation, nurses are at the frontline and have frequent contact with HIV/AIDS positive patients. Their counselling behaviour is influenced by their knowledge about counselling as well as their beliefs, attitudes and perceptions about barriers to counselling (Mntlangula, Khuzwayo and Taylor 2017, 52).

The lack of health workers is a major bottleneck in implementing evidence-based interventions, thus providing a significant challenge to meeting the Sustainable Development Goals for 2030 (UNFPA 2014). Midwifery encompasses a range of functions that span care of women during pregnancy, labour, and the postpartum period, as well as care of the newborn (UNFPA 2014). Thus, midwives are expected to meet the demands of their ever-expanding roles, such as counselling, while working under difficult conditions, such as with pregnant women who are HIV positive. They have to do this without adequate quality pre-service and in-service education, medical supplies, and in some cases without support from nurse managers (WHO 2016).

Worldwide, the importance of the protocol on HIV/AIDS testing, counselling and structures in the workplace is one of the most critical debates in health care circles. The professional and legal duties of midwives require them to care for pregnant women who are HIV/AIDS positive. Midwives are expected by their employers, the South African Nursing Council (SANC) as well as the World Health Organization (WHO) to be constantly informed about any new developments regarding quality care provided to patients by midwives (DoH 2013). Provider initiated testing and counselling for pregnant women should be considered a routine component of the antenatal package (WHO 2013, 43) in order to identify at-risk patients as soon as possible.

\section{Research Problem}

In 2010, South Africa's policy on voluntary counselling and testing (VCT) for HIV/AIDS was revised to require that this service be offered by health providers on the occasion of any patient's visit to any health facility for any ailment (SANAC Secretariat 2010). The PMTCT Programme not only includes ARV intervention, but also entails VCT, HIV/AIDS testing, revised obstetric practices, and infant feeding practices. However, achieving these goals requires that midwives should be well-prepared and supported by nurse managers in their service delivery especially in view of the complexities of HIV/AIDS prevention, the expansion of their role, and treatment and care (Crowley 2015). At departmental meetings, the midwives at an antenatal unit were complaining of being unable to scale up their competencies to follow the protocol in HIV/AIDS counselling. They indicated that the nurse managers did not support them in becoming competent to effectively counsel patients. It seemed as if the midwives had 
minimal quality pre-service and in-service education regarding this protocol, and lacked support from their supervisors (nurse managers) when questions around the protocol arise. It was, therefore, unclear how the midwives experienced the support of the nurse managers in complying with the mandated HIV/AIDS testing and counselling protocol (antenatal and postnatal). The findings could inform the development of workplace strategies to support midwives in their role of acting as counsellors to HIV/AIDS patients.

\section{Purpose of the Study}

The purpose of the study was to investigate the gap in nurse managers' support to midwives in implementing HIV/AIDS testing and counselling within protocol. The objectives of the study were to:

- explore the experiences of midwives in implementing HIV/AIDS testing and counselling; and

- describe the support of nurse managers to midwives in implementing HIV/AIDS testing and counselling.

\section{Research Methodology}

\section{Study Design}

The study followed an exploratory, descriptive, contextual, qualitative design. Qualitative research enquires an in-depth exploration into the meaning which individuals ascribe to a social or human problem, such as support from managers (Creswell and Poth 2017). The collection of data was done in a natural setting in which the services were rendered to patients. The research was exploratory in nature to gain insight into midwives' experiences of implementing HIV/AIDS testing and counselling at an antenatal clinic. Descriptive research was used to collect accurate data on the specific phenomenon. The contextual departure of the research referred to the specific event of support for counselling in the "naturalistic setting" (Grove, Burns and Gray 2015) of an antenatal clinic. An in-depth description of the phenomenon in the particular clinic was given.

\section{Population and Sample}

A target population is the entire population in which the researcher is interested (Polit and Beck 2017). The study population was the professional midwives placed in an antenatal clinic in the Central Region of Cape Town, Western Cape, South Africa.

The researcher used purposive, subjective sampling and relied on her own judgment when she chose patients from the target population to participate in the study. The eligibility criteria stipulated to include participants who were: 
- working in the antenatal clinic for a year or longer;

- involved with testing and counselling pregnant HIV/AIDS positive women;

- readily available to participate in the study; and

- registered with the SANC as professional midwives.

Interviews were conducted with eight participants who met the inclusion criteria, and the number of participants was determined by data saturation, when redundancy of information was observed, and no new information came to the fore (Polit and Beck 2017).

\section{Data Collection}

For the study, the researcher used method triangulation, through semi-structured individual interviews with an interview schedule and descriptive field notes to develop a comprehensive understanding of the phenomenon. Probing was used to gain an indepth understanding of the experiences shared with the interviewer. The researcher and participants agreed upon a scheduled time to conduct the interviews. The interviews took around 45 minutes in a private room at the antenatal clinic in the research setting. While conducting the interviews, the researcher digitally recorded the data obtained from the experiences shared by the participants. Probing was used to follow up on questions posed; to gain an in-depth understanding of the participants' views; and to cover every aspect in as much depth as possible. As the data was collected, it was analysed in order to establish the point of data saturation.

\section{Data Analysis}

The transcription of the data from the interviews and field notes was done collectively, thus the data was triangulated to obtain more in-depth results and conclusions. Data triangulation is the use of multiple sources of referents to draw conclusions about what constitutes the truth (Fusch, Fusch and Ness 2018, 19). Triangulation provided corroborating evidence for validating the accuracy of the study (Creswell and Poth 2017, 204). Open coding was used to organise data collected in the individual interviews. The researcher and separately an independent coder analysed the data, after which a consensus meeting was held to ensure accuracy of the interpretation of the themes and categories. The independent coder was experienced in qualitative data analysis.

In analysing the data, the researcher and independent coder carefully listened to the digital recordings and simultaneously made notes about the context and variations in the participants/midwives' experiences in implementing HIV/AIDS testing and counselling at the clinic. The researcher read and re-read the transcripts to get an overview and understanding of the data. Similar topics were grouped together and the most descriptive wording for the topics was identified to be used as themes. The 
abbreviations for the categories were arranged alphabetically in codes and marked in the margins of the transcripts. The data in each category was collated and analysed.

\section{Trustworthiness}

Van der Walt, Brink and Van Rensburg $(2018,126)$ describe "trustworthiness" as a means of measuring the research relevance, methodological consistency and general fairness of the qualitative research method of data collection and analysis.

"Credibility" was maintained through prolonged engagement with the participants in order to understand their in-depth views regarding the six semi-structured research questions. The researcher implemented purposive sampling, used detailed data collection methods and conducted data triangulation. Credibility was further enhanced through the use of thick descriptions about the data gathered (Maree 2017, 123).

"Confirmability" was ensured during the data collection by taking descriptive field notes and digitally recording the interviews. The independent coder and researcher agreed on the interpretation of the themes and categories after the data analysis. A pilot individual interview was conducted to explore whether any adjustments to the interview guide were necessary, but none were required.

"Transferability" refers to the results of the qualitative research being applicable to other contexts with other participants (Maher et al. 2018, 1). The researcher provided a full and purposeful account of the context, participants and research design of the study, so that readers can make their own decision about the usefulness for a similar setting (Maree 2017, 124).

Maree $(2017,124)$ claims that "dependability" in qualitative research is demonstrated through the research design and its implementation; data gathering; and reflective appraisal of the project. The researcher took field notes during the interviews; was consistent in data collection; provided a detailed description of how the data was collected, analysed and recorded; and kept the records for audit purposes.

\section{Ethical Considerations}

Ethical approval was obtained from the ethics committee of the Faculty of Community and Health Sciences at a university in the Western Cape, the Western Cape Department of Health $(\mathrm{DoH})$, and the head of the selected antenatal clinic. Furthermore, the participants each signed an informed written consent form. The researcher invited the participants to participate in the study, and gave them an information sheet on the purpose and process of the study. Each interview was conducted in a private room for no longer than 45 minutes. No name of any participant appeared on the records or transcripts. The participants were assured of confidentiality around their personal details and that they could withdraw at any stage of the research process. 
Results

\section{Participants' Profiles}

None of the eight participants had previously undergone the available but not yet compulsory training in HIV/AIDS offered by the DoH. However, all the participants were currently working with HIV/AIDS patients visiting the clinic. In presenting the findings, the verbatim quotes will indicate the participants who were labelled P1 to P8.

Table 1: Participants' profiles

\begin{tabular}{|l|l|l|l|l|}
\hline $\begin{array}{l}\text { Participant } \\
(\boldsymbol{n}=\mathbf{8})\end{array}$ & Age & Gender & Level of education & $\begin{array}{l}\text { Years of experience } \\
\text { working at the clinic }\end{array}$ \\
\hline P1 & 28 & Female & Degree & 6 \\
\hline P2 & 28 & Female & Degree & 3 \\
\hline P3 & 38 & Female & Diploma & 14 \\
\hline P4 & 36 & Female & Degree & 12 \\
\hline P5 & 24 & Female & Degree & 2 \\
\hline P6 & 26 & Male & Degree & 3 \\
\hline P7 & 46 & Female & Diploma & 20 \\
\hline P8 & 29 & Female & Degree & 5 \\
\hline
\end{tabular}

The findings indicated the themes and categories, including the participants' voices; the researcher's reflexivity; and the interpretation of the problem (Creswell and Poth 2017). Three main themes emerged from the data analysis, namely: the lack of support provided to midwives; the protocol midwives should follow during counselling of patients; and the emotional difficulty of HIV/AIDS testing and counselling (see Table 2).

Table 2: Themes and categories

\begin{tabular}{|c|c|}
\hline Theme & Category \\
\hline $\begin{array}{l}\text { Lack of support provided to } \\
\text { midwifes providing HIV/AIDS } \\
\text { counselling }\end{array}$ & $\begin{array}{l}\text { - Lack of emotional support from the nurse } \\
\text { manager } \\
\text { - } \text { Challenges around staffing and workload } \\
\text { - } \quad \text { Need for more training opportunities } \\
\text { - } \quad \text { Emotional difficulty to provide counselling }\end{array}$ \\
\hline $\begin{array}{l}\text { Protocol midwives should follow } \\
\text { during counselling of patients }\end{array}$ & $\begin{array}{l}\text { - Mandate to do HIV/AIDS testing and } \\
\text { counselling } \\
\text { - Struggling to implement protocol in HIV/AIDS } \\
\text { counselling } \\
\text { - Lack of visibility of protocol }\end{array}$ \\
\hline
\end{tabular}




\section{Theme 1: Lack of Support Provided to Midwives Giving HIV/AIDS Counselling}

Nurse managers perform management functions to deliver health care to patients and offer support to their subordinates. The support that nurse managers provide to their subordinates, such as midwives, includes the provision of encouragement. In the study, the participants gave accounts of how they felt lack of support from the nurse managers.

\section{Lack of Emotional Support from Nurse Managers}

Employers should provide opportunities for psychological support (e.g. debriefing sessions) to nurses and midwives working with HIV/AIDS positive clients (DENOSA 2019). It appeared that in some midwives' experiences they were not adequately supported by their nurse managers with regard to the emotional effects of conducting HIV/AIDS testing and counselling:

On the issue of nurse managers' support to midwives ... midwives need emotional support ... because it is not just the patient who must be supported when she finds out that she is positive ... the midwife too needs emotional support after informing the patient. (P1)

I would not say that there is support given to midwives by nurse managers ... because everything is referred to the sister who is involved in the HIV/AIDS testing and counselling ... so from them (managers) directly ... they do not give support. (P2)

It has been found that nurses are attending to more patients than their workload allows, thereby putting them under pressure to see as many patients as possible (Mntlangula, Khuzwayo and Taylor 2017).

Trust is essential in working relationships because employees depend on each other for help in difficult situations. This would certainly be ruined if, for example, a manager involved in a trustworthy relationship, let the midwives down (Kutsyuruba and Walker 2016).

Participant 8 indicated the need for emotional support from the nurse managers, as she experienced guilt and felt displeased with the different reasons for why the patients were not being well informed about HIV/AIDS:

Some of the patients don't even get proper counselling ... you just assume that the doctor did speak to the patient ... the doctor just said to the patient that the patient should be tested ... the patient gives the consent ... you come as a person doing the test ... after the test you rely on the doctor to come back ... and half of the time they don't come back to provide that proper counselling that the patient is supposed to get. (P8)

Participant 5 lacked emotional backing while having a heavy workload and she was upset that the patients received insufficient HIV/AIDS counselling: 
... but the involvement with the patient self ... there is a lack in that ... and like we as nurses ... we don't give the patient that support (counselling) because we don't have the time to sit with that patient. (P5)

The Democratic Organisation for Nurses in South Africa (DENOSA 2019) states that managers have a responsibility of periodically providing psychological support to allow for debriefing by nurses who deal with HIV/AIDS issues.

\section{Challenges around Staffing and Workload}

The issue of being short staffed in midwifery practice is detrimental to the quality of care that clients receive. Participant 8 was of the view that the scarcity of staff in a midwifery facility impacted negatively on patient care, and that every midwife on duty had different ways of grappling with issues of staff shortage:

There is one person in the facility that I'm working in ... who is responsible for HIV/AIDS issues. It is too much for one person ... and that person is not available on night shifts and public holidays or weekends. (P8)

Participant 5 mentioned that more staff assistance was needed to address the needs of patients:

We do have an operational manager that is assigned for HIV/AIDS only ... she's working with the counsellors but she is not really involved with the patients. (P5)

The proportion of nurses to patients can affect the staff's mental and emotional health as well as their productivity in the workplace, which then determines the patients' overall health (Gutsan et al. 2018).

\section{Need for More Training Opportunities}

In the study, the midwives felt that they were not being given enough training opportunities to empower them to increase their knowledge in HIV/AIDS counselling: Participant 1 indicated that although she received training, it was inadequate:

As a midwife I am not trained to properly counsel the patient ... so I find that as a challenge ... because if you do not have the real knowledge about counselling the patients ... how can you support the patient. (P1)

According to participant 6 , inadequate training could be caused by the lack of reenforcement courses due to new developments in HIV/AIDS:

We don't get re-enforcement courses on HIV/AIDS testing and counselling ... and we as midwives need that course .... as a reminder of how to do everything. (P6)

Participant 6 further indicated that she only received brief and inadequate training: 
Once someone come to give a crash course on the HIV/AIDS testing and counselling ... but that was not enough because I think you should understand it ... you can't focus on something partially and expect us to implement that afterwards ... it is not fair. (P6)

It seemed that participant 3 was told about and gained information concerning different counselling sessions that should be offered to patients; however, they were not implemented in the clinic:

... because I am aware that there should be a pre-counselling session and a postcounselling session ... we don't have that exposure to those things ... so we can't give adequate counselling to the patient ... and follow-up. (P3)

In addition to lack of training opportunities, some midwives felt discriminated against in terms of accessing available training opportunities. The participants felt that the nurse managers showed favouritism and prejudice in allowing certain staff members to attend training. Participant 1 said:

I also want to add that not all the midwives are sometimes allowed to attend courses as there are just some who are specifically chosen to attend those courses. I feel that this is unfair and all midwives need training on all levels and all should be given the privilege to be educated. (P1)

Motivation is a key ingredient in building an effective, successful staff component in a health organisation (Jooste 2017). It seemed that the individual training needs of staff were not identified for them to be sent on relevant courses. According to participant 8:

Even when there is in-service training ... they will not motivate their staff and say, do this training. They don't sit with the subordinates and go through the possible courses and what is the risk or the advantages of doing certain courses with their staff members ... maybe they can improve on that. (P8)

However, it was not clear from the interviews whether this practice was intentional on the part of the nurse managers or if it was a feeling of discrimination among the midwives.

\section{Theme 2: Protocol Midwives Should Follow during Counselling of Patients}

Counselling protocol involves the counsellor seeing a person (e.g. a patient) in a confidential setting to investigate problems that the client is experiencing in life (Bradford Grammar School 2018). Most protocols are binding on employees as they usually relate to the management of consumers with urgent, possibly life-threatening, conditions. Midwifery protocols and policies are fundamental to the midwifery profession and practice. 


\section{The Mandate for Providing HIV/AIDS Counselling}

Many health care organisations and institutions have their own unique set of protocols that all staff must follow. The study participants experienced that it was sometimes challenging to follow protocol. According to participant 7:

Not mandated to do HIV/AIDS testing and counselling. (P7)

In certain instances, the study indicated that the midwives might feel that they were not mandated to do certain tasks. They were, however, aware that adhering to protocols in general could improve the results in the work environment. Following protocols in midwifery practice may enhance the ongoing provision of quality care for clients. However, following protocols in midwifery practice seemed to have become a heavy burden. On the one hand, it prevented midwives from applying their personal and professional judgments. On the other hand, it seemed that the protocols expected midwives to perform tasks outside of their normal daily practice.

A few participants suggested that other team members should be responsible for conducting the HIV/AIDS testing and counselling at the antenatal clinic:

It is supposed to be done by the interns ... so they are supposed to do the testing, counselling and giving the result to the patient ... in my experience ... HIV/AIDS testing and counselling is not really directed to us ... we do not have that platform where we are so involved in that. (P2)

The midwife is not really much involved in that process ... it is just the counsellors that are there ... conducting the testing and counselling ... so we are not really there to observe how the patient is ... throughout the process. (P4)

The midwives wanted to feel that the duties they performed were in alignment with their scope of practice. This would enable them to mirror the types of client for whom they need to care and the appropriate procedures and activities they needed to perform (Jooste 2017). However, the participants seemed to have a lack of exposure to HIV/AIDS testing and counselling at the antenatal clinic that prevented them from providing adequate training in HIV/AIDS disclosure for health care workers as well as the provision of standardised disclosure materials (Kalembo et al. 2018).

\section{Struggling to Implement Protocol in HIV/AIDS Counselling}

It is appropriate that persons authorised by law to practise nursing care are accountable for their actions and must adhere to legal, practice and ethical standards (Jooste 2017). Whenever there is a need in an antenatal clinic to do HIV/AIDS testing and counselling of clients, nurses should feel that they are mandated to do it. The study participants felt that the clinic times could hamper adequate HIV/AIDS counselling. According to participant 5: 
We do have our own counsellors in the clinic on a daily basis ... but there is nobody over weekends ... if we do get un-booked patients over the weekends then it is a worry because then the midwife has to do the counselling and the doctor ... the doctors maybe. (P5)

The participants described the difficulty of sometimes having no other choice but to act on and implement certain procedures as directives from the doctor:

... and I am not too keen on doing HIV/AIDS testing and counselling either ... it is only during the weekends when we as midwives are forced to do it. (P6)

... so normally we are only asked to do the HIV/AIDS testing ... and on odd occasions when there's weekends or maybe on public holidays when the HIV/AIDS counsellors don't work. (P8)

It became evident that there was an unclear scope of how counselling skills in HIV/AIDS and testing should be used and who should use them.

\section{Lack of Visibility of Protocol}

Lack of visibility simply means an insufficiency, a shortage, or an absence of something required or desired. The lack of the visibility of protocols in the antenatal clinic was also frustrating for the midwives. Protocols are guidelines to follow to ensure that quality care is provided to clients and to cover midwives in the event of complications arising. Guidelines should be readily visible and accessible to staff in order for them to do their work properly. The study participants felt that this was not the case:

It is a bit of a challenge because the protocols are not visible on the walls ... as a midwife I am not trained to properly counsel the patient ... so I find that as a challenge ... because if you do not have the real knowledge about counselling the patient ... how can you support the patient ... with the protocols. (P1)

When it comes to protocols ... there are no visuals with regard to protocols. (P2)

Protocols are procedures for a well-organised antenatal unit. It is a need to provide standardised informative materials, such as a protocol on HIV/AIDS disclosure, for health care workers to ensure quality patient care (Kalembo et al. 2018).

\section{Emotional Difficulty in Providing Counselling}

Due to the intensity and the associated stigma of HIV/AIDS, the participants indicated that it was emotionally difficult to talk about the subject. It affects almost everyone in some way and not everybody is at liberty to speak about the subject. In the case of the midwives in the study, conveying the results of the HIV/AIDS test and counselling the patient were emotionally difficult duties to perform: 
Emotionally it is too much for you as midwife ... to give the patient all that information $\ldots$ it was not a nice experience. (P1)

I don't want to be the one who tells the patient that she is HIV/AIDS positive ... I do not like it ... it is not for me. (P6)

\section{Discussion}

The study explored nurse managers' support to midwives in implementing HIV/AIDS testing and counselling within protocol. Client support is the support and care that need to be provided to patients by midwives in an antenatal unit. Holistically, an approach to client care involves all client care aspects, such as the environment, equipment and health care professionals, not just the individual client (Minnaar 2016, 84). Nurse managers' support to midwives could affect the quality of counselling midwives provide to patients with HIV/AIDS. These factors include lack of emotional support from nurse managers to midwives in following protocol; lack of personal and professional development opportunities created for midwives; and staffing challenges that nurse managers face on a daily basis to assist midwives in client support within protocol. Although the study highlighted the nurse managers' support role with the midwives, it indicated that poor interpersonal relationships could translate to shortcomings in patient-centred care. Patient-centred care with a focus on effective interpersonal communication offers opportunities for staff, such as midwives in health systems, to improve the quality of care (Waweru et al. 2019).

Midwives need emotional support from nurse managers in their role of counselling HIV/AIDS patients. Fear and anxiety among midwives in carrying out screening and counselling could result in a lack of care and counselling to HIV/AIDS patients. Wellplanned preparation of staff development opportunities should be available to ensure that midwives understand their role and unique contributions in offering counselling services. This should be complemented with the support of managers, in moderating the emotional involvement of midwives with patients to help mitigate the sense of failure amongst them and to prevent burnout (Busza et al. 2018).

Nurses spend almost all their time communicating with patients. Verbal and non-verbal messages that pass between midwives and patients have therapeutic or non-therapeutic qualities and outcomes. In HIV/AIDS the combination of patients with complex needs, together with the demands of busy nursing staff, can produce a less than favourable therapeutic environment where communication is blocked by various barriers (WHO 1993), such as lack of protocols. It would appear that improving relations between midwives and nurse managers can help midwives pay more attention to the "anxiety spectrum" HIV/AIDS patient may be facing. Nurses need to attend to patients' physical, psychological, emotional and spiritual needs. 
In the interviews, the midwives expressed their worries that they were neither being completely informed about training opportunities nor adequately staffed. Work environments where communication is neither open nor transparent could breed mistrust among workmates. Though it is imperative that the right number and type of nurses should be available at the right time and place to undertake the work required to fulfil the organisation's goals (Sokhela 2018, 96), in resource-limited communities, this remains a huge challenge. However, the study acknowledges that nursing care in antenatal settings has certain set standards that require professional nurses, to have specific competencies in their speciality of midwifery. Because knowledge about HIV/AIDS treatment is evolving, the provision of ongoing counselling training and education in the process of care remains critical. For example, the diagnosis of HIV/AIDS positive results is in itself traumatic, with the affected people dealing with the possibility of many changes, losses and adjustments, often in situations of social isolation or poverty (WHO 1993). Discussions about HIV/AIDS testing and counselling between midwives and patients are never easy and midwives should be skilled to execute such a difficult task. The study indicated that midwives either did not feel competent in a counselling role on HIV/AIDS or that other members of the multidisciplinary team fulfilled this role. Conversations about HIV/AIDS between midwives and patients were lacking. It could be more effective if midwives with counselling skills were used to explore specific issues for individuals and were up to date with relevant counselling issues such as new treatment or management.

The study confirmed that ongoing education, coaching and mentoring in HIV/AIDS counselling need to be offered to midwives. Their important role in counselling should be emphasised as part of their daily duties, for which they could receive constructive feedback (Nkozi 2016, 256). Continuous short courses or sessions for midwives in HIV/AIDS counselling could promote trust and staff responsibility and accountability for effective patient care outcomes. Well-informed health care providers should treat patients with dignity, respect and sensitivity to cultural values and expressed needs. This may include recognising the needs of supporting the family in counselling regarding the HIV/AIDS status of their family member. As family members may serve as carers, they should be guided in decision-making processes about the essential aspects around the care of the HIV/AIDS patient.

A mandate is when a conclusive set of rules are followed to conduct a certain task. It is also included in an employee's scope of practice. The employer lays down a mandate for a job that an employee needs to do. The scope of practice is defined as the activities that a professional nurse performs in the delivery of client care (Jooste 2017). According to Gulaid (2015), midwives are accountable practitioners whose relationships with their patients are inclusive of giving support and advice during and after pregnancy. As such midwives are expected to fulfil their roles of coordinating and planning ongoing treatment and services after the patient has been diagnosed as HIV/AIDS positive. 


\section{Recommendations}

The following recommendations were made for practice, further educational practices and research: In the "midwifery practice" context, nurse managers should adequately reflect the required level of midwifery skills and responsibilities that can link from appointment and orientation up till the evaluation of performances. Developmental opportunities created by nurse manager can create an environment of openness and trust, in which midwives can approach nurse managers on challenges experience. A developmental approach should be followed in units, with nurse managers as advocates who should have a plan to address midwives' educational needs on a continuous basis. The latest in protocols related to health and nursing legislation, policies and regulations in midwifery should be discussed. This gathering could serve as a professional support network of knowledge management enhancing an informed health care delivery environment. All midwives should also take responsibility and should be held accountable for practising within their scope of practice, as they are registered with the regulatory body. The scope of practice stipulates treating and counselling all patients towards a healthy life. Nurse managers should monitor the effectiveness of midwivespatient communication, to determine the essential ways through which midwives can interact with patients. These could include the availability of important HIV/AIDS information, such as videos playing in the waiting area.

For further educational practices, midwives should be encouraged to join professional associations and international organisations that distribute important counselling information on websites. Nurse managers should create a learning environment (e.g. notice board) to make midwives aware of national and international workshops, conferences and forums organised regarding midwifery practices and counselling. Another possibility is for nurse managers to establish a yearly workshop for midwives in the same geographical area to attend and discuss matters such as the protocol to follow in HIV/AIDS counselling. At such a workshop, national developments and barriers that midwifery personnel face, can be addressed and debated in order to improve the quality of midwifery practices. Continuing education opportunities can also include instructional in-service programmes and the availability of knowledgeable peers (Jooste 2017).

Research in HIV/AIDS is vast; however, more research can be conducted regarding the patients' own role in dealing with the treatment of the disease.

\section{Limitations}

The study was limited in two main areas. Firstly, the sample size was small and mostly females. However, with more males entering the nursing profession, their experiences could enrich the insight into the support of nurse managers to midwives in implementing HIV/AIDS testing and counselling. Secondly, the findings could not be generalised to another facility as only one antenatal clinic was included in the qualitative study. 


\section{Conclusion}

Discussions about HIV/AIDS and related topics can arouse strong feelings of uncertainty, hurt, fear and lack of self-worth amongst patients. At the same time, even the trained midwives show the need for clarity about their role and responsibilities in counselling and supporting patients as it also becomes emotionally difficult for them to deal with the patients' HIV/AIDS diagnosis. The midwives indicated that the nurse managers should be supported when they are confronted with emotionally difficult situations relating to HIV/AIDS testing and counselling.

\section{References}

Avert. 2019a. "Global Information and Education on HIV/AIDS and AIDS." Accessed September 23, 2019. https://www.avert.org/professionals/hivprogramming/prevention/prevention-mother-child

Avert. 2019b. "Prevention of Mother-to-Child Transmission (PMTCT) of HIV." Accessed September 24, 2019. https://www.avert.org/professionals/hivprogramming/prevention/prevention-mother-child

Bradford Grammar School. 2018. "Counselling Protocol and Practice.” Accessed September 30, 2019. https://www.bradfordgrammar.com/wp-content/uploads/2018/09/BGSCounselling-Protocol-and-Practice.pdf

Busza, J., E. Dauya, T. Bandason, V. Simms, C. D. Chikwari, M. Makamba, G. Mchugh, S. Munyati, P. Chonzi, and R. A. Ferrand. 2018. "The Role of Community Health Workers in Improving HIV/AIDS Treatment Outcomes in Children: Lessons Learned from the ZENITH Trial in Zimbabwe." Health Policy and Planning 33 (3): 328-334. https://doi.org/10.1093/heapol/czx187

Campbell, O. M. R., C. Calvert, A. Testa, M. Strehlow, L. Benova, E. Keyes, F. Donnay, D. Macleod, S. Gabrysch, L. Rong, C. Ronsmans, S. Sadruddin, M. Koblinsky, and P. Bailey. 2016. The Lancet Maternal Health Series: The Scale, Scope, Coverage, and Capability of Childbirth Care. London: London School of Hygiene and Tropical Medicine. https://doi.org/10.1016/S0140-6736(16)31528-8

Creswell, J. W., and C. N. Poth. 2017. Qualitative Inquiry and Research Design: Choosing among Five Approaches. London: Sage.

Crowley, T. 2015. "Trends in Task Shifting in HIV Treatment in Africa: Effectiveness, Challenges and Acceptability to the Health Professions." African Journal of Primary Health Care and Family Medicine 7 (1): 1-9. https://doi.org/10.4102/phcfm.v7i1.807

DENOSA (Democratic Organisation for Nurses in South Africa). 2019. "Denosa HIV/AIDS Position Papers." Accessed September 28, 2019. https://www.denosa.org.za/Documents_View.php?id=354 
DoH (Department of Health). 2013. The National Strategic Plan for Nurse Education, Training and Practice 2012/13-2016/17. Pretoria: DoH.

Fusch, P., G. E. Fusch, and L. R. Ness. 2018. “Denzin’s Paradigm Shift: Revisiting Triangulation in Qualitative Research.” Journal of Social Change 10 (1): 19-32.

Grove, S. K., N. Burns, and J. R. Gray. 2015. Understanding Nursing Research: Building an Evidence-Based Practice. New York: Elsevier Health Sciences.

Gulaid, L. A. 2015. Community-Facility Linkages to Support the Scale Up of Lifelong Treatment for Pregnant and Breastfeeding Women Living with HIV. Uganda: UNICEF.

Gutsan, E., J. Patton, W. K. Willis, and A. Coustasse-Hencke. 2018. "Burnout Syndrome and Nurse-to-Patient Ratio in the Workplace." Paper presented at the 54th Annual MBAA Conference, Chicago, Illinois, April 18-20.

Jooste, K. 2017. The Principles and Practice of Nursing and Health Care: Ethos and Professional Practice, Management, Staff Development and Research. Pretoria: Van Schaik.

Kalembo, F. W., G. E. Kendall, M. Ali, and A. F. Chimwaza. 2018. "Healthcare Workers' Perspectives and Practices Regarding the Disclosure of HIV/AIDS Status to Children in Malawi: A Cross-Sectional Study." BMC Health Services Research 18 (1): a540. https://doi.org/10.1186/s12913-018-3354-9

Kutsyuruba, B., and K. D. Walker. 2016. "The Destructive Effects of Distrust: Leaders as Brokers of Trust in Organizations." In The Dark Side of Leadership: Identifying and Overcoming Unethical Practice in Organizations, edited by A. H. Noremore and J. S. Brooks, 133-154. London: Emerald. https://doi.org/10.1108/S1479-366020160000026008

Maher, C., M. Hadfield, M. Hutchings, and A. Eyto. 2018. "Ensuring Rigor in Qualitative Data Analysis: A Design Research Approach to Coding Combining NVivo with Traditional Material Methods.” International Journal of Qualitative Methods 17 (1): 1-13.

Maree, K. 2017. First Steps in Research. 2nd ed. Pretoria: Van Schaik.

Mbombo, N., and K. Jooste. 2016. "International, Regional and National Health Priorities and Legislation on Maternal Health and a Role of a Specialist Midwife as the Key Implementer." In Healthcare Dynamics for the Specialist Professional Nurse, edited by K. Jooste. Kenwyn: Juta.

Minnaar, A. 2016. “The Management Process: An Overview." In Healthcare Dynamics for the Specialist Professional Nurse, edited by K. Jooste. Kenwyn: Juta. 
Mntlangula, M. N., N. Khuzwayo, and M. Taylor. 2017. "Nurses' Perceptions about Their Behavioural Counselling for HIV/AIDS, STIs and TB in eThekwini Municipality Clinics KwaZulu-Natal, South Africa." Health SA Gesondheid 22 (1): 52-60. https://doi.org/10.1016/j.hsag.2016.09.001

Nkozi, Z. "Challenges in Nursing Practice." In Healthcare Dynamics for the Specialist Professional Nurse, edited by K. Jooste. Kenwyn: Juta.

Phetlhu, D. R., R. M. M. Bimerew, R. Marie-Modeste, M. Naidoo, and J. Igumbor. 2018. "Nurses' Knowledge of Tuberculosis, HIV, and Integrated HIV/TB Care Policies in Rural Western Cape, South Africa." Journal of the Association of Nurses in AIDS Care 29 (6): 876-886.

Polit, D. F., and C. T. Beck. 2017. Essentials of Nursing Research: Appraising Evidence for Nursing Practice. 8th ed. Philadelphia: Lippincott Williams and Wilkins.

SANAC (South African National AIDS Council) Secretariat. 2010. The National HIV/AIDS Counselling and Testing Campaign Strategy. Pretoria: SANAC.

Scheffler, R., J. Campbell, G. Cometto, A. Maeda, J. Liu, T. A. Bruckner, D. R. Arnold, and T. Evans. 2018. "Forecasting Imbalances in the Global Health Labor Market and Devising Policy Responses." Human Resources for Health 16 (1): a5.

Tagutanazvo, O. B., A. G. W. Nolte, and A. Temane. 2019. "Experiences of Women Enrolled in a Prevention of Mother to Child Transmission of Human Immunodeficiency Virus Infection Programme in Zimbabwe." Health SA Gesondheid 24: a1088. https://doi.org/10.4102/hsag.v24i0.1088

UNAIDS (Joint United Nations Programme on HIV/AIDS). 2019. "Sub-Saharan Africa Sees Reduction of HIV among Children." Accessed January 3, 2019. https://africa.cgtn.com/2019/07/17/unaids-sub-saharan-africa-sees-reduction-of-hivamong-children/

UNFPA (United Nations Population Fund). 2014. “The State of the World's Midwifery 2014: A Universal Pathway. A Woman's Right to Health.” Accessed March 10, 2020. https://www.unfpa.org/sowmy

Van der Walt, C., H. I. L. Brink, and G. van Rensburg. 2018. Fundamentals of Research Methodology for Healthcare Professionals. 4th ed. Kenwyn: Juta.

Waweru, E., N. D. P. Sarkar, F. Ssengooba, M. Gruénais, J. Broerse, and B. Criel. 2019. "Stakeholder Perceptions on Patient-Centred Care at Primary Health Care Level in Rural Eastern Uganda: A Qualitative Inquiry." Accessed October 4, 2019. https://journals.plos.org/plosone/article?id=10.1371/journal.pone.0221649

WHO (World Health Organization). 1993. HIV/AIDS Prevention and Care: Teaching Modules for Nurses and Midwives. Geneva: WHO. 
WHO (World Health Organization). 2013. WHO Nursing and Midwifery Progress Report 2008-2012. Geneva: WHO.

WHO (World Health Organization). 2016. WHO and Partners Call for Better Working Conditions for Midwives. Geneva: WHO. 